TRANSACTIONS OF THE

AMERICAN MATHEMATICAL SOCIETY

Volume 354, Number 3, Pages 975-992

S 0002-9947(01)02837-9

Article electronically published on September 19, 2001

\title{
SOME COMBINATORICS OF BINOMIAL COEFFICIENTS AND THE BLOCH-GIESEKER PROPERTY FOR SOME HOMOGENEOUS BUNDLES
}

\author{
MEI-CHU CHANG
}

\begin{abstract}
A vector bundle has the Bloch-Gieseker property if all its Chern classes are numerically positive. In this paper we show that the non-ample bundle $\Omega_{\mathbb{P}_{n}}^{p}(p+1)$ has the Bloch-Gieseker property, except for two cases, in which the top Chern classes are trivial and the other Chern classes are positive. Our method is to reduce the problem to showing, e.g. the positivity of the coefficient of $t^{k}$ in the rational function $\frac{(1+t)\left(\begin{array}{l}n \\ p\end{array}\right)(1+3 t)\left(\begin{array}{c}n \\ p-2\end{array}\right) \cdots(1+(p-1) t)\left(\begin{array}{c}n \\ 2\end{array}\right)(1+(p+1) t)}{(1+2 t)\left(\begin{array}{c}n \\ p-1\end{array}\right)(1+4 t)\left(\begin{array}{c}n \\ p-3\end{array}\right) \cdots(1+p t)\left(\begin{array}{c}n \\ 1\end{array}\right)}$ (for $p$ even).
\end{abstract}

The existence (or nonexistence) of low rank vector bundles on the projective $n$-space has been intriguing to many mathematicians $([\mathrm{BH}],[\mathrm{E} 1],[\mathrm{EHS}],[\mathrm{H} 2],[\mathrm{H} 3$, $[\mathrm{Ho},[\mathrm{HM}], \mathrm{KPR}, \mathrm{LV}, \overline{\mathrm{OSS}},[\mathrm{T},[\mathrm{V}], \underline{\mathrm{Z}}])$. For $n \geq 4$, the only known bundles of rank $r<n$, besides the Horrocks-Mumford bundle (of rank 2 on $\mathbb{P}^{4}$ ) [HM], and the Horrocks bundle (of rank 3 on $\mathbb{P}^{5}$ ) [Ho, are the null-correlation bundles OSS, and Tango's example of rank $n-1$ indecomposable bundles on $\mathbb{P}^{n}$ T]. A nullcorrelation bundle is the quotient of $\Omega_{\mathbb{P}_{n}}(2)$ by $\mathcal{O}_{\mathbb{P}_{n}}$, for $n$ odd. Tango's example is the quotient of $\Omega_{\mathbb{P}_{n}}^{n-2}(n-1)$ by the right number of sections. Here, $\Omega_{\mathbb{P}_{n}}$ is the cotangent bundle, and $\Omega_{\mathbb{P}_{n}}^{n-2}$ is its $(n-2)$ nd exterior power. The idea behind the construction of the null-correlation bundle and Tango's example is very simple: for a globally generated bundle of rank $r \geq n$, and with trivial top Chern class, its quotient by $r-n+1$ sections is a vector bundle. This same idea was also used in C] to characterize Buchsbaum bundles. Along these lines, a natural question to ask is

Does $\Omega_{\mathbb{P}_{n}}^{p}(p+1)$ have trivial top Chern class?

Since $\Omega_{\mathbb{P}_{n}}^{p}(p+1)$ is generated by global sections and its Chern classes are nonnegative, this is the same question as

Is $c_{n} \Omega_{\mathbb{P}_{n}}^{p}(p+1)$ positive?

A theorem that immediately comes to mind is by Bloch and Gieseker [BG].

If $E$ is an ample vector bundle of rank $r$ on an $n$-dimensional variety, and if $r>n$, then all Chern classes of $E$ are numerically positive.

So we give the following

Definition. A vector bundle has the Bloch-Gieseker property if all its Chern classes are numerically positive.

Received by the editors September 10, 2000.

2000 Mathematics Subject Classification. Primary 14F05; Secondary 14J60, 05A10.

(C)2001 American Mathematical Society 
However, the theorem of Bloch and Gieseker does not apply to $\Omega_{\mathbb{P}_{n}}^{p}(p+1)$, because of the following observation by Z. Ran.

Observation. $\Omega_{\mathbb{P}_{n}}^{p}(p+1)$ is not ample.

In fact, the restriction of $\Omega_{\mathbb{P}_{n}}^{p}(p+1)$ on a line has trivial summands.

Using some fun combinatorics of binomial coefficients, we show that with a few exceptions, the Chern classes of $\Omega_{\mathbb{P}_{n}}^{p}(p+1)$ are all positive.

Theorem. The homogeneous bundle $\Omega_{\mathbb{P}_{n}}^{p}(p+1)$ is not ample and it has the BlochGieseker property, except for the following cases:

(i) $p=1$ and $n$ is odd,

(ii) $p=n-2$.

In the two cases above, the top Chern classes are trivial and the other Chern classes are positive

We note that in the theorem above, case (i) gives the null-correlation bundle, and case (ii) gives Tango's example.

Fulton and Lazarsfeld [FL generalized Bloch and Gieseker's result and showed that the Schur polynomials for ample bundles are positive. So the next question is

Question. Are the Schur polynomials for $\Omega_{\mathbb{P}_{n}}^{p}(p+1)$ positive?

S. Katz and S. Stromme have written computer software [KS to compute the numerical invariants we are interested for given projective spaces.

We also note that L. Ein [E2] has a nice argument to show the vanishing of $c_{n} \Omega_{\mathbb{P}_{n}}^{n-2}(n-1)$ (which is the same as the Segre class of $\left.\Omega_{\mathbb{P}_{n}}(2)\right)$ by looking at the map to the Grassmannian of lines given by the tautological line bundle. The fibre of this map is $\mathbb{P}_{1}$.

Problem. Find a geometric argument to show that $\Omega_{\mathbb{P}_{n}}^{p}(p+1)$ is positive (zero).

Our method does not give an immediate answer to the question. Our approach is to estimate the coefficients of the Chern polynomial of $\Omega_{\mathbb{P}_{n}}^{p}(p+1)$. Recall that the Chern polynomial is the polynomial whose coefficient of $t^{k}$ is the $k$ th Chern class. It is multiplicative for exact sequences [H1].

We use the Koszul resolution of $\Omega_{\mathbb{P}_{n}}^{p}(p+1)$ to write the Chern polynomial of $\Omega_{\mathbb{P}_{n}}^{p}(p+1)$ as the quotient of the products of Chern polynomials $(1+a t)^{N}$ of $\stackrel{N}{\oplus} \mathcal{O}_{\mathbb{P}_{n}}(a)$. Hence the problem is reduced to determining whether certain functions of the binomial coefficients are positive. More precisely, we let

$$
\Omega^{p}(p+1):=\Omega_{\mathbb{P}_{n-1}}^{p}(p+1) .
$$

The Koszul resolution of $\Omega^{p}(p+1)$ is

$$
0 \rightarrow \Omega^{p}(p+1) \rightarrow\left(\begin{array}{c}
n \\
p
\end{array}\right) \mathcal{O}(1) \rightarrow\left(\begin{array}{c}
n \\
p-1
\end{array}\right) \mathcal{O}(2) \rightarrow \cdots \rightarrow n \mathcal{O}(p) \rightarrow \mathcal{O}(p+1) \rightarrow 0 .
$$

Therefore the $k$ th Chern class of $\Omega^{p}(p+1)$ is the coefficient of $t^{k}$ in the rational function

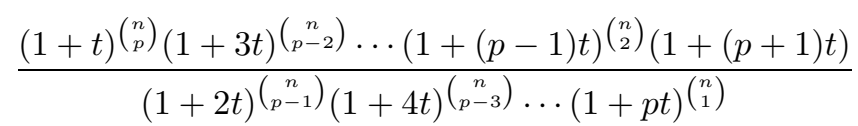

(for $p$ even), 


$$
\frac{(1+t)^{\left(\begin{array}{c}
n \\
p
\end{array}\right)}(1+3 t)^{\left(\begin{array}{c}
n \\
p-2
\end{array}\right)} \cdots(1+(p-2) t)^{\left(\begin{array}{c}
n \\
3
\end{array}\right)}(1+p t)^{\left(\begin{array}{c}
n \\
1
\end{array}\right)}}{(1+2 t)^{\left(\begin{array}{c}
n \\
p-1
\end{array}\right)}(1+4 t)^{\left(\begin{array}{c}
n \\
p-3
\end{array}\right)} \cdots(1+(p-1) t)^{\left(\begin{array}{c}
n \\
2
\end{array}\right)}(1+(p+1) t)}
$$

(for $p$ odd).

Our idea is to write (2) as a product of power series with positive coefficients. Therefore, we first give a criterion for the coefficient of $t^{k}$ in

$$
\frac{(1+a t)^{M}}{[(1+(a-1) t)(1+(a+1) t)]^{N}}, \quad a>0,
$$

to be positive (Proposition 2). Then we apply the criterion to as many factors as possible in the numerator in (2).

Note that when $a=1,(3)$ is

$$
\frac{(1+t)^{M}}{(1+2 t)^{N}}
$$

and the criterion takes care of the beginning quotient in $(2)$ and $\left(2^{\prime}\right)$.

This argument works for $p \leq \frac{n}{2}$, when the binomial coefficient $\left(\begin{array}{l}n \\ k\end{array}\right)$ as a function of $k$, is increasing for $k \leq p$. The ideal situation is when there are not enough factors in the denominator in (2), i.e. when, as a function of $k,\left(\begin{array}{l}n \\ k\end{array}\right)$ is concave up. So, in order to apply Proposition 2 we multiply the deficit to both the denominator and the numerator. Hence we end up with a product of rational functions (3), with $a=1,3,5, \cdots, p-1$, and nonnegative powers of $(1+2 t),(1+4 t), \cdots$ (see expressions (10), (11) and (13)). However, $\left(\begin{array}{l}n \\ k\end{array}\right)$ is concave down for $\frac{n}{2}-\sqrt{n}<k<\frac{n}{2}+\sqrt{n}$ and the denominator does have unused powers of $(1+(p-k+1) t)$ for $k$ in this range. So we give a criterion for the positivity of the coefficient of $t^{k}$ in the "left-over" from function (3).

$$
\frac{\prod\left(1+a_{i} t\right)^{M_{i}}}{\prod\left(1+b_{j} t\right)^{N_{j}}}, \quad a_{i}>b_{j}, \forall i, j,
$$

to take care of the negative powers of $(1+2 t),(1+4 t), \cdots$ (see expression (13)).

For $p>\frac{n}{2}$, we note that if

$$
Q_{q}:=\Omega_{\mathbb{P}_{n-1}}^{q}(q+1),
$$

then

$$
Q_{q}^{*}(1)=Q_{n-1-q} .
$$

Dualizing exact sequence (1) and twisting by $\mathcal{O}_{\mathbb{P}_{n-1}}(1)$, we get

$$
\begin{aligned}
0 \rightarrow & \mathcal{O}(-q) \rightarrow n \mathcal{O}(-q+1) \rightarrow \cdots \rightarrow\left(\begin{array}{c}
n \\
q-1
\end{array}\right) \mathcal{O}(-1) \rightarrow\left(\begin{array}{c}
n \\
q
\end{array}\right) \mathcal{O} \rightarrow Q_{q}^{*}(1) \\
& =Q_{n-1-q} \rightarrow 0 .
\end{aligned}
$$

Hence, if

$$
p:=n-1-q>\frac{n}{2}
$$

then

$$
n>2 q+2 .
$$

Hence the $k$ th Chern class of

$$
Q_{p}=Q_{n-1-q}=Q_{q}^{*}(1)
$$


is the coefficient of $t^{k}$ in the rational function

$$
\frac{(1-2 t)^{\left(\begin{array}{c}
n \\
q-2
\end{array}\right)}(1-4 t)^{\left(\begin{array}{c}
n \\
q-4
\end{array}\right)} \cdots(1-q t)}{(1-t)^{\left(\begin{array}{c}
n \\
q-1
\end{array}\right)}(1-3 t)^{\left(\begin{array}{c}
n \\
q-3
\end{array}\right)} \cdots(1-(q-1) t)^{\left(\begin{array}{c}
n \\
1
\end{array}\right)}}
$$

(for $q$ even),

$$
\frac{(1-2 t)^{\left(\begin{array}{c}
n \\
q-2
\end{array}\right)}(1-4 t)^{\left(\begin{array}{c}
n \\
q-4
\end{array}\right)} \cdots(1-(q-1) t)^{\left(\begin{array}{c}
n \\
1
\end{array}\right)}}{(1-t)^{\left(\begin{array}{c}
n \\
q-1
\end{array}\right)}(1-3 t)^{\left(\begin{array}{c}
n \\
q-3
\end{array}\right)} \cdots(1-(q-2) t)^{\left(\begin{array}{c}
n \\
2
\end{array}\right)}(1-q t)}
$$

(for $q$ odd).

We call this the negative case, (because of the negative coefficients in the factors) and the case for $p \leq \frac{n}{2}$ the positive case. In some sense, the negative case is the dual of the positive case. However, one result does not follow immediately from the other.

Similar to the criteria for the positivity of the coefficients of $t^{k}$ in the rational functions (3) and (4), we have criteria for those in

$$
\frac{[(1-(a-1) t)(1-(a+1) t)]^{N}}{(1-a t)^{M}}
$$

and

$$
\frac{\prod\left(1-a_{i} t\right)^{N_{i}}}{\prod\left(1-b_{j} t\right)^{M_{j}}}, \quad a_{i}<b_{j}, \forall i, j .
$$

Note again that in (7), when $a=1$, this is

$$
\frac{(1-2 t)^{N}}{(1-t)^{M}} \text {. }
$$

We need it for the beginning quotient in $(6)$ and $\left(6^{\prime}\right)$.

The paper is organized in the following way:

In Section 1, we treat the positive case.

In Section 2, we treat the negative case.

We use $[r]$ for the largest integer $n$ such that $n \leq r$.

Acknowledgements. The author would like to thank L. Ein, S. Nollet, and Z. Ran for helpful communications.

\section{The POSITIVe CASE}

In this section we first give criteria for the positivity of the coefficients of $t^{k}$ in the rational functions (3) and (4). Then we use them to prove the positivity for function (2), and outline the difference for $\left(2^{\prime}\right)$.

Lemma 1. Let $c(M, N, k)$ be the coefficient of $t^{k}$ in

$$
\frac{(1+a t)^{M}}{[(1+(a-1) t)(1+(a+1) t)]^{N}} .
$$

Then we have the following properties

(i) $c(M, N, 0)=1$, $c(M, N, 1)=(M-2 N) a$.

(ii) $c(M, 0, k)= \begin{cases}\left(\begin{array}{c}M \\ k\end{array}\right) a^{k}, & \text { if } M \geq k, \\ 0, & \text { otherwise. }\end{cases}$ 
(iii) $c(M, N, k)=c(M-2, N-1, k)+c(M-2, N, k-2)$.

Proof. (i) and (ii) are obvious.

Since the value of $a$ does not play any role in our argument, we write the case $a=1$ here for simpler notation.

Property (iii) follows from the following identity

$$
\begin{aligned}
\frac{(1+t)^{M}}{(1+2 t)^{N}} & =\frac{(1+t)^{M-2}}{(1+2 t)^{N-1}}\left(\frac{1+2 t}{1+2 t}+\frac{t^{2}}{1+2 t}\right) \\
& =\frac{(1+t)^{M-2}}{(1+2 t)^{N-1}}+\frac{(1+t)^{M-2}}{(1+2 t)^{N}} t^{2}
\end{aligned}
$$

Proposition 2. For $k>1$, the coefficient of $t^{k}$ in

$$
\frac{(1+a t)^{M}}{[(1+(a-1) t)(1+(a+1) t)]^{N}}
$$

is positive, if

$$
M-2 N \geq k-1 \text {. }
$$

Proof. It is by induction. Here we give the details of the steps to show where the hypothesis comes from.

We use the notation as in Lemma 1.

Lemma 1 (iii) gives

$$
\begin{aligned}
c(M, N, k)= & c(M-2, N-1, k)+c(M-2, N, k-2) \\
= & c(M-4, N-2, k)+2 c(M-4, N-1, k-2)+(c(M-4, N, k-4) \\
= & c(M-6, N-3, k)+3 c(M-6, N-2, k-2) \\
& +3 c(M-6, N-1, k-4)+c(M-6, N, k-6)
\end{aligned}
$$

After $i$ steps, $c(M, N, k)$ is a linear combination of $c(M-2 i, \bar{N}, \bar{k})$, where the possible pairs of $(\bar{N}, \bar{k})$ are

$$
(N-i, k),(N-i+1, k-2), \cdots,(N-1, k-2 i+2),(N, k-2 i) .
$$

Our goal is to do reduction until a term has $\bar{N}$ or $\bar{k}$ equal 0 (or $\bar{k}$ equal 1 , if $k$ is odd), then we put it in the "positive number" term and continue applying Lemma 1 (iii) to the other terms. So in this situation, we say

(*) After $i$ steps, $c(M, N, k)$ is the sum of a positive number and a linear combination of $c(M-2 i, \bar{N}, \bar{k})$.

and discuss the possible $(\bar{N}, \bar{k})$.

Case 1. $N \geq \frac{k}{2}$.

(a) $k=2 i$, an even number. After $i$ steps, for $c(M-k, \bar{N}, \bar{k})$ the possible $(\bar{N}, \bar{k})$ are

$$
(N-i, k),(N-i+1, k-2), \cdots,(N-2,4),(N-1,2) .
$$

Note that $c(M-k, N, 0)$ was put in the "positive number" term. 
We continue $N-i$ more steps, the possible $(\bar{N}, \bar{k})$ for $c(M-2 N, \bar{N}, \bar{k})$ are

$$
(1, k-2),(2, k-4), \cdots,(i-2,4),(i-1,2) \text {. }
$$

Again, $c(M-2 N, 0, k)$ and $c(M-2 N, i, 0)$ are put in the "positive number" term.

Finally, another $i-1$ step reduce those not in "positive number" term to $c(M-2 N-k+2,1,0)$ or $c(M-2 N-k+2,0,2)$, which is nonnegative.

To assure all steps work, the condition is $M-2 N-k+2 \geq 0$.

(b) $k=2 i+1$, an odd number. The steps are the same. In $(*)$, "positive number" is replaced by "nonnegative number". However, at least one of them i.e. $c(M-2 N, 0, k)$ is positive. To be sure that we have nonnegative coefficients put in the "nonnegative number" term at the $i$ th step, we want $c(M-2 i, N, 1)=$ $M-(k-1)-2 N \geq 0$.

Case 2. $\frac{k}{2}>N$.

The argument is similar. We outline the case when $k$ is even here.

After $N$ steps, the possible $(\bar{N}, \bar{k})$ for $c(M-2 N, \bar{N}, \bar{k})$ are

$$
(N, k-2 N),(N-1, k-2 N+2), \cdots,(2, k-4),(1, k-2) .
$$

Another $\frac{k}{2}-N$ steps give

$$
(N-1,2),(N-2,4), \cdots,(4,2 N-4),(2,2 N-2)
$$

as possible $(\bar{N}, \bar{k})$ for $c(M-k, \bar{N}, \bar{k})$.

It is clear that $N-1$ more steps finish the proof and the condition is $M-k-$ $2(N-1) \geq 0$.

Remark 2.1. To use this proposition, when $M, k$ are given we take $N=\left[\frac{M-k+1}{2}\right]$. Therefore

$$
N=\left[\frac{1}{2} M-\frac{n}{2}+1\right]
$$

works for all $k \leq n-1$.

Lemma 3. Let $c(M, N, k)$ be the coefficient of $t^{k}$ in

$$
\frac{(1+a t)^{M}}{(1+b t)^{N}}, \quad a>b
$$

Then we have the following

(i) $c(M, N, 0)=1$,

$c(M, N, 1)=a M-b N$.

(ii) $c(M, 0, k)=\left\{\begin{array}{l}\left(\begin{array}{c}M \\ k\end{array}\right) a^{k}, \text { if } M \geq k, \\ 0, \quad \text { otherwise. }\end{array}\right.$

(iii) $c(M, N, k)=c(M-1, N-1, k)+(a-b) c(M-1, N, k-1)$.

Proof of (iii). This follows from

$$
\begin{aligned}
\frac{(1+a t)^{M}}{(1+b t)^{N}} & =\frac{(1+a t)^{M-1}}{(1+b t)^{N-1}}\left(\frac{1+b t}{1+b t}+\frac{(a-b) t}{1+b t}\right) \\
& =\frac{(1+a t)^{M-1}}{(1+b t)^{N-1}}\left(1+\frac{(a-b) t}{(1+b t)}\right) .
\end{aligned}
$$


Proposition 4. For $k>1$, the coefficient of $t^{k}$ in

$$
\frac{(1+a t)^{M}}{(1+b t)^{N}}, \quad a>b
$$

is positive, if

$$
M-N \geq k-1 .
$$

Proof. This is similar, but simpler than the proof of Proposition 2. We use the language as in $(*)$.

After $i$ steps, $c(M, N, k)$ is a linear combination of $c(M-i, \bar{N}, \bar{k})$, where the possible $(\bar{N}, \bar{k})$ are

$$
(N, k-i),(N-1, k-i+1), \cdots,(N-i+1, k-1),(N-i, k) .
$$

Assuming $N \geq k$.

After $k$ steps, we get to $c(M-k, \bar{N}, \bar{k})$, with $(\bar{N}, \bar{k})$.

$$
(N-1,1),(N-2,2), \cdots,(N-k+1, k-1),(N-k, k) .
$$

Another $N-k$ steps give $c(M-N, \bar{N}, \bar{k})$ with $(\bar{N}, \bar{k})$

$$
(k-1,1),(k-2,2), \cdots,(2, k-2),(1, k-1) .
$$

The last $k-1$ steps finish the proof.

The case $k>N$ is similar.

Proposition 5. The coefficient of $t^{k}$ is positive in

$$
\frac{\prod\left(1+a_{i} t\right)^{M_{i}}}{\prod\left(1+b_{j} t\right)^{N_{j}}}, \quad a_{i}>b_{j}, \forall i, j
$$

if

$$
\sum M_{i}-\sum N_{j} \geq k-1
$$

Proof. Let $a=\min _{i}\left\{a_{i}\right\}$ and let $b=\max _{j}\left\{b_{j}\right\}$. Then the coefficient of $t^{k}$ in $\frac{\prod\left(1+a_{i} t\right)^{M_{i}}}{\prod\left(1+b_{j} t\right)^{N_{j}}}$ is at least that in $\frac{(1+a t)^{\sum M_{i}}}{(1+b t)^{\sum N_{j}}}$ (see Remark 5.1 for reasons).

For $k>1$, this follows from Proposition 4 .

For $k=1$, it is because

$$
a \sum M_{i}-b \sum N_{j}>b\left(\sum M_{i}-\sum N_{j}\right) \geq \sum M_{i}-\sum N_{j} \geq k-1 .
$$

Remark 5.1. Let $a=\min _{i}\left\{a_{i}\right\}$ and let $b=\max _{j}\left\{b_{j}\right\}$. Then the coefficient of $t^{k}$ in $\frac{\prod\left(1+a_{i} t\right)^{M_{i}}}{\prod\left(1+b_{j} t\right)^{N_{j}}}$ is at least that in $\frac{(1+a t)^{\sum M_{i}}}{(1+b t)^{\sum N_{j}}}$, if $a>b$ and $\sum M_{i}-\sum N_{j} \geq k-1$.

Proof. Let $a_{1}=\max _{i}\left\{a_{i}\right\}$ and $b_{1}=\min _{j}\left\{b_{j}\right\}$. As in the proof of Lemma 3, we write

$$
\begin{aligned}
\frac{\prod\left(1+a_{i} t\right)^{M_{i}}}{\prod\left(1+b_{j} t\right)^{N_{j}}}= & \frac{\left(1+a_{1} t\right)^{M_{1}-1}}{\left(1+b_{1} t\right)^{N_{1}-1}} \frac{\prod_{i>1}\left(1+a_{i} t\right)^{M_{i}}}{\prod_{i>1}\left(1+b_{j} t\right)^{N_{j}}} \\
& +\left(a_{1}-b_{1}\right) \frac{\left(1+a_{1} t\right)^{M_{1}-1} \prod_{i>1}\left(1+a_{i} t\right)^{M_{i}}}{\prod\left(1+b_{j} t\right)^{N_{j}}} t .
\end{aligned}
$$


By induction, the coefficient of $t^{k}$ is at least that in

$$
\frac{(1+a t)^{\sum M_{i}-1}}{(1+b t)^{\sum N_{j}-1}}+\left(a_{1}-b_{1}\right) \frac{(1+a t)^{\sum M_{i}-1}}{(1+b t)^{\sum N_{j}}} t .
$$

By the notation in Lemma 3, this is

$$
c\left(\sum M_{i}-1, \sum N_{j}-1, k\right)+\left(a_{1}-b_{1}\right) c\left(\sum M_{i}-1, \sum N_{j}, k-1\right),
$$

which is at least

$$
\begin{array}{r}
c\left(\sum M_{i}-1, \sum N_{j}-1, k\right)+(a-b) c\left(\sum M_{i}-1, \sum N_{j}, k-1\right) \\
=c\left(\sum M_{i}, \sum N_{j}, k\right),
\end{array}
$$

the coefficient of $t^{k}$ in $\frac{(1+a t)^{\sum M_{i}}}{(1+b t)^{\sum N_{j}}}$.

The initial step is either Proposition 4 or Lemma 3(i) and hypothesis.

Theorem 6. The coefficient of $t^{k}$ in

$$
\prod_{\ell=0}^{p}(1+(\ell+1) t)^{(-1)^{\ell}\left(\begin{array}{c}
n \\
p-\ell
\end{array}\right)}
$$

is positive for $n \geq 2 p+1$ and $p \geq 3$.

Proof. First we assume $p$ is even. Then our rational function is expression (2).

To use Remark 2.1, we define

$$
N(r):=\left[\frac{1}{2}\left(\begin{array}{l}
n \\
r
\end{array}\right)-\frac{n}{2}+1\right] .
$$

Then expression (2) becomes

$$
\frac{(1+t)^{\left(\begin{array}{l}
n \\
p
\end{array}\right)}}{(1+2 t)^{N(p)}} \frac{(1+3 t)^{\left(\begin{array}{c}
n \\
p-2
\end{array}\right)}}{[(1+2 t)(1+4 t)]^{N(p-2)}} \cdots \frac{(1+(p-1) t)^{\left(\begin{array}{c}
n \\
2
\end{array}\right)}}{[(1+(p-2) t)(1+p t)]^{N(2)}} \cdot P(t)
$$

where

$$
\begin{gathered}
P(t)=(1+2 t)^{N(p)+N(p+2)-\left(\begin{array}{c}
n \\
p-1
\end{array}\right)}(1+4 t)^{N(p-2)+N(p-4)-\left(\begin{array}{c}
n \\
p-3
\end{array}\right) \ldots} \\
(1+p t)^{N(2)-\left(\begin{array}{c}
n \\
1
\end{array}\right)}(1+(p+1) t) .
\end{gathered}
$$

We define

$$
E(r):=N(r+2)+N(r)-\left(\begin{array}{c}
n \\
r+1
\end{array}\right) .
$$

Note that

$$
2 E(r)=\left(\begin{array}{c}
n \\
r+2
\end{array}\right)+\left(\begin{array}{l}
n \\
r
\end{array}\right)-2\left(\begin{array}{c}
n \\
r+1
\end{array}\right)-2(n-2)-\varepsilon,
$$

where $\varepsilon \in\{0,1,2\}$.

It is clear that there is an even integer $p_{0}<\frac{n}{2}$ such that

$$
E(r) \geq 0 \text { if and only if } r \leq p_{0} .
$$


If $p-2 \leq p_{0}$, then $P(t)$ is a polynomial and we are done. Otherwise, using our new notation, (11) becomes

(13)

$$
\begin{aligned}
P(t)= & (1+2 t)^{E(p-2)}(1+4 t)^{E(p-4)} \cdots \\
& (1+(p-2) t)^{E(2)}(1+p t)^{N(2)-n}(1+(p+1) t) \\
= & \frac{\left(1+\left(p-p_{0}\right) t\right)^{E\left(p_{0}\right)} \cdots(1+(p-2) t)^{E(2)}(1+p t)^{N(2)-n}(1+(p+1) t)}{(1+2 t)^{-E(p-2)}(1+4 t)^{-E(p-4)} \cdots\left(1+\left(p-p_{0}-2\right) t\right)^{-E\left(p_{0}+2\right)}} .
\end{aligned}
$$

According to Proposition 5, the condition for the coefficient of $t^{k}$ in $P(t)$ to be positive for $k \leq n-1$ is

$$
\sum_{p_{0} \geq \underset{\text { even }}{r} \geq 2} E(r)+N(2)-n+1-\left(-\sum_{p-2 \geq \underset{\text { even }}{r} \geq p_{0}+2} E(r)\right) \geq n-2
$$

i.e.

$$
\sum_{p-2 \geq_{\text {even }} \geq 2} E(r)+N(2) \geq 2 n-3 .
$$

According to (9) and (12), it suffices to show

$$
\begin{aligned}
& \sum_{p-2 \geq_{\text {even }} \geq 2}\left[\left(\begin{array}{c}
n \\
r+2
\end{array}\right)+\left(\begin{array}{c}
n \\
r
\end{array}\right)-2\left(\begin{array}{c}
n \\
r+1
\end{array}\right)\right] \\
& \quad-(n-2)(p-2)-(p-2)+\left(\begin{array}{c}
n \\
2
\end{array}\right)-n+1 \geq 4 n-6 .
\end{aligned}
$$

The next lemma and simplification reduce it further to

$$
\left(\begin{array}{c}
n-1 \\
p
\end{array}\right)-\left(\begin{array}{l}
n-1 \\
p-1
\end{array}\right) \geq(p+1)(n-1)-2
$$

or

$$
\frac{(n-1) !}{p !(n-p) !}(n-2 p) \geq(p+1)(n-1)-2 .
$$

For $n \geq 2 p+1$ and $p \geq 6$, the left hand side is at least

$$
\begin{aligned}
\frac{(n-1)(n-2) \cdots(n-p+1)}{2 \cdot 3 \cdots p} & \geq \frac{(n-1)(n-2)(n-3)}{4 \cdot 5 \cdot 6} \frac{(n-4)(n-5)}{2 \cdot 3} \\
& \geq n(n-1) \quad(\text { if } n \geq 14) \\
& >(p+1)(n-1)-2 .
\end{aligned}
$$

For $p=6$ and $n=13$, one checks inequality (16) directly.

The case $p$ odd is similar. Instead of (14), (16), (17) we have

$$
\begin{gathered}
\sum_{p-2 \geq \underset{\text { odd }}{r} \geq 1} E(r) \geq n-2 \\
\frac{(n-1) !}{p !(n-p) !}(n-2 p) \geq(p+2)(n-1)-3,
\end{gathered}
$$




$$
\begin{aligned}
\frac{(n-1)(n-2) \cdots(n-p+1)}{2 \cdot 3 \cdots p} & \geq \frac{(n-1)(n-2)(n-3)(n-4)}{4 \cdot 5 \cdot 6 \cdot 7} \frac{(n-5)(n-6)}{2 \cdot 3} \\
& \geq(n+1)(n-1) \\
& >(p+2)(n-1)-3,
\end{aligned}
$$

when $n \geq 2 p+1, p \geq 7$ (hence $n \geq 15$ ).

For $p=3$ (respectively 4,5 ), we check that inequality (14) or $\left(14^{\prime}\right)$ holds for $n \geq 10$ (resp. 10,11), and the remaining few cases directly from (2).

Lemma 7. (i) Let $p$ be even, then

$$
\begin{aligned}
& \sum_{p-2 \geq_{\text {even }} \geq 2}\left(\begin{array}{c}
n \\
r+2
\end{array}\right)+\left(\begin{array}{c}
n \\
r
\end{array}\right)-2\left(\begin{array}{c}
n \\
r+1
\end{array}\right) \\
& =\left(\begin{array}{c}
n-1 \\
p
\end{array}\right)-\left(\begin{array}{c}
n-1 \\
p-1
\end{array}\right)+(n-1)-\left(\begin{array}{c}
n-1 \\
2
\end{array}\right) .
\end{aligned}
$$

(ii) Let $p$ be odd, then

$$
\sum_{p-2 \geq r \begin{array}{l}
\text { odd } \\
\text { en }
\end{array}}\left(\begin{array}{c}
n \\
r+2
\end{array}\right)+\left(\begin{array}{c}
n \\
r
\end{array}\right)-2\left(\begin{array}{c}
n \\
r+1
\end{array}\right)=\left(\begin{array}{c}
n-1 \\
p
\end{array}\right)-\left(\begin{array}{c}
n-1 \\
p-1
\end{array}\right)+1-\left(\begin{array}{c}
n-1 \\
1
\end{array}\right) .
$$

Proof of (i). The left hand side is

$$
\begin{aligned}
\left(\begin{array}{l}
n \\
2
\end{array}\right) & +2\left[\left(\begin{array}{l}
n \\
4
\end{array}\right)-\left(\begin{array}{l}
n \\
3
\end{array}\right)+\left(\begin{array}{l}
n \\
6
\end{array}\right)-\left(\begin{array}{l}
n \\
5
\end{array}\right)+\cdots+\left(\begin{array}{c}
n \\
p-2
\end{array}\right)-\left(\begin{array}{c}
n \\
p-3
\end{array}\right)\right] \\
& +\left(\begin{array}{c}
n \\
p
\end{array}\right)-2\left(\begin{array}{c}
n \\
p-1
\end{array}\right) .
\end{aligned}
$$

Using $\left(\begin{array}{l}n \\ r\end{array}\right)=\left(\begin{array}{c}n-1 \\ r\end{array}\right)+\left(\begin{array}{c}n-1 \\ r-1\end{array}\right)$ to simplify the above expression, we conclude the proof.

The proof of (ii) is identical.

The next theorem takes care of the cases $p=1$ and $p=2$.

Theorem 8. The coefficient of $t^{k}$ is positive for $k \leq n-1$ in the following expression

(i) $\left(p=1\right.$ case) $\frac{(1+t)^{n}}{(1+2 t)}$, except for $k=n-1$ and $n$ even.

(ii) $\left(p=2\right.$ case) $\frac{(1+t)\left(\begin{array}{l}n \\ 2\end{array}\right)(1+3 t)}{(1+2 t)^{n}}$.

Proof. (i) The positivity follows from Lemma 1 and Proposition 2 (for $a=1$ ). Another way to look at this rational function is as the product

$$
\left(1+\left(\begin{array}{c}
n \\
1
\end{array}\right) t+\left(\begin{array}{c}
n \\
2
\end{array}\right) t^{2}+\cdots\right)\left(1-2 t+2^{2} t^{2}-\cdots\right)
$$

The coefficient of $t^{n-1}$ is

$$
\begin{aligned}
c(n)= & \left(\begin{array}{c}
n \\
n-1
\end{array}\right)-2\left(\begin{array}{c}
n \\
n-2
\end{array}\right)+2^{2}\left(\begin{array}{c}
n \\
n-3
\end{array}\right)-\cdots \\
& +(-1)^{n-2} 2^{n-2}\left(\begin{array}{c}
n \\
1
\end{array}\right)+(-1)^{n-1} 2^{n-1} .
\end{aligned}
$$


Since

$$
\begin{gathered}
(-1)^{n-1} 2 c(n)=(2-1)^{n}-(-1)^{n}, \\
c(n)=0 \text { if and only if } n \text { is even. }
\end{gathered}
$$

(ii) Proposition 2 implies that $\frac{(1+t)\left(\begin{array}{c}n \\ 2\end{array}\right)}{(1+2 t)^{n}}$ has positive coefficient of $t^{k}$ for $k \leq n-1$, if $n \geq 7$. The remaining cases are easy to check by hand.

\section{The Negative CASE}

We first give criteria for the positivity of the coefficient of $t^{k}$ in rational functions (7) and (8). Then we prove this for (6) and outline the difference for $\left(6^{\prime}\right)$.

Lemma 9. Let $c(N, M, k)$ be the coefficient of $t^{k}$ in

$$
\frac{[(1-(a-1) t)(1-(a+1) t)]^{N}}{(1-a t)^{M}} .
$$

Then we have the following properties

(i) $c(N, M, 0)=1$,

$$
c(N, M, 1)=(M-2 N) a .
$$

(ii) $c(0, M, k)=\left(\begin{array}{c}M+k-1 \\ k\end{array}\right) a^{k}$,

(iii) $c(N, M, k)=c(N-1, M-2, k)-c(N-1, M, k-2)$.

Proof. (i) is clear. For (ii) we use Taylor expansion of $f(t)=(1-a t)^{-M}$. The coefficient of $t^{k}$ is

$$
\frac{f^{(k)}(0)}{k !} a^{k}=\frac{M(M+1) \cdots(M+k-1)}{k !} a^{k}=\left(\begin{array}{c}
M+k-1 \\
k
\end{array}\right) a^{k} .
$$

(iii) As before, since the reasoning is the same, we write the case $a=1$ for easier reading.

$$
\frac{(1-2 t)^{N}}{(1-t)^{M}}=\frac{(1-2)^{N-1}}{(1-t)^{M-2}}\left(1-\frac{t^{2}}{(1-t)^{2}}\right)=\frac{(1-2 t)^{N-1}}{(1-t)^{M-2}}-\frac{(1-2 t)^{N-1}}{(1-t)^{M}} t^{2} .
$$

Proposition 10. Let $c(N, M, k)$ be the coefficient of $t^{k}$ in

$$
\frac{[(1-(a-1) t)(1-(a+1) t)]^{N}}{(1-a t)^{M}} .
$$

For $k>1$, if

$$
a^{2}(M-2 N+1)(M-2 N)>N k(k-1),
$$

then

$$
0<c(N, M, k)<a^{k}\left(\begin{array}{c}
M-2 N+k-1 \\
k
\end{array}\right) .
$$


Proof. Lemma 9 (iii) implies

$$
\begin{aligned}
c(N, M, k) & =c(N-1, M-2, k)-c(N-1, M, k-2) \\
& =c(N-2, M-4, k)-c(N-2, M-2, k-2)-c(N-1, M, k-2) \\
& \vdots \\
& =c(0, M-2 N, k)-\sum_{s=0}^{N-1} c(N-s-1, M-2 s, k-2) .
\end{aligned}
$$

We will use induction. First, we notice that if $N, M, k$ satisfy assumption (18), then so do $N-s-1, M-2 s, k-2$. Now, we check the initial step in the induction.

For $k=2$,

$$
\begin{aligned}
c(N, M, 2) & =c(0, M-2 N, 2)-\sum_{s=0}^{N-1} c(N-s-1, M-2 s, 0) \\
& =a^{2}\left(\begin{array}{c}
M-2 N+1 \\
2
\end{array}\right)-N .
\end{aligned}
$$

For $k=3$,

$$
\begin{aligned}
c(N, M, 3) & =c(0, M-2 N, 3)-\sum_{s=0}^{N-1} c(N-s-1, M-2 s, 1) \\
& =a^{3}\left(\begin{array}{c}
M-2 N+2 \\
3
\end{array}\right)-N(M-2 N+2) a \\
& =(M-2 N+2) a\left(a^{2} \frac{(M-2 N+1)(M-2 N)}{3 \cdot 2}-N\right) .
\end{aligned}
$$

Both satisfy the inequalities.

The induction argument is identical to the two cases above.

Remark 10.1. To optimize the use of this proposition, when $N, k$ are given, we take

$$
M=\left[2 N+\frac{\sqrt{N}}{a} k\right]+1
$$

and when $M, k$ are given, we take

$$
N=\left[\frac{M}{2}-\frac{k}{2 a} \sqrt{\frac{M}{2}}\right] .
$$

Lemma 11. The coefficient of $t^{k}$ in

is positive, if

$$
\frac{(1-a t)^{N}}{(1-b t)^{M}}, \quad 0<a<b,
$$

$$
M \geq N
$$

Proof. This is because

$$
\frac{(1-a t)^{N}}{(1-b t)^{M}}=\left(1+\frac{(b-a)}{(1-b t)} t\right)^{N} \frac{1}{(1-b t)^{M-N}},
$$

and both terms in the right hand side have positive coefficients in their expansions. 
Proposition 12. The coefficient of $t^{k}$ in

is positive, if

$$
\frac{\prod\left(1-a_{i} t\right)^{N_{i}}}{\prod\left(1-b_{j} t\right)^{M_{j}}} \quad \text { with } a_{i}<b_{j}, \forall i, j
$$

$$
\sum N_{i} \leq \sum M_{j}
$$

Proof. It suffices to show that the positivity of the coefficients of

$$
\frac{(1-a t)^{\sum N_{i}}}{(1-b t)^{\sum M_{j}}}
$$

implies that of $\frac{\prod\left(1-a_{i} t\right)^{N_{i}}}{\prod\left(1-b_{j} t\right)^{M_{j}}}$. Here $a=\max \left\{a_{i}\right\}$, and $b=\min \left\{b_{j}\right\}$. This follows from the following two facts.

Assuming that $\frac{(1-a t)^{N}}{(1-b t)^{M}}$ has positive coefficients,

(i) if $a^{\prime}<a$, then so does $\frac{\left(1-a^{\prime} t\right)^{N}}{(1-b t)^{M}}$,

(ii) if $b^{\prime}>b$, then so does $\frac{(1-a t)^{N}}{\left(1-b^{\prime} t\right)^{M}}$.

These are because

(i) $\frac{\left(1-a^{\prime} t\right)^{N}}{(1-b t)^{M}}=\frac{(1-a t)^{N}}{(1-b t)^{M}}\left(1+\frac{\left(a-a^{\prime}\right) t}{(1-a t)}\right)^{N}$,

(ii) $\frac{(1-a t)^{N}}{\left(1-b^{\prime} t\right)^{M}}=\frac{(1-a t)^{N}}{(1-b t)^{M}}\left(1+\frac{\left(b^{\prime}-b\right) t}{\left(1-b^{\prime} t\right)}\right)^{N}$.

Theorem 13. The coefficient of $t^{k}$ in

$$
\prod_{\ell=1}^{q}(1-\ell t)^{(-1)^{\ell}\left(\begin{array}{c}
n \\
q-\ell
\end{array}\right)}
$$

is positive for $n \geq 2 q+2$ and $q \geq 4$.

Proof. (i) For $q \geq 6$ even, our rational function is expression (6). We want to write the function as a product of rational functions with positive coefficient for $t^{i}$ for $i \leq k$. Most of these functions are in the form of (7). So we apply Remark 10.1 to as many factors in the denominator of (6) as possible. We define

$$
N(r):=\left[\frac{1}{2}\left(\begin{array}{l}
n \\
r
\end{array}\right)-\frac{(n-1)}{2 \sqrt{2}(q-r)}\left(\begin{array}{l}
n \\
r
\end{array}\right)^{1 / 2}\right] .
$$

(Note that $N(r) \geq 0$ for $2 \leq r<\frac{n}{2}$.)

Then (6) becomes

$$
\begin{aligned}
& \frac{(1-2 t)^{N(q-1)}}{(1-t)^{\left(\begin{array}{c}
n \\
q+1
\end{array}\right)}} \frac{[(1-2 t)(1-4 t)]^{N(q-3)}}{(1-3 t)^{\left(\begin{array}{c}
n \\
q-3
\end{array}\right)}} \cdots \\
& \quad \frac{[(1-(q-4) t)(1-(q-2) t)]^{N(3)}}{(1-(q-3) t)^{\left(\begin{array}{c}
n \\
3
\end{array}\right)}} \cdot P_{1}(t) P_{2}(t),
\end{aligned}
$$

where

$$
\begin{aligned}
P_{1}(t) & =(1-2 t)^{\left(\begin{array}{c}
n \\
q-2
\end{array}\right)-N(q-1)-N(q-3)}(1-4 t)^{\left(\begin{array}{c}
n \\
q-4
\end{array}\right)-N(q-3)-N(q-5)} \\
& \cdots(1-(q-4) t)^{\left(\begin{array}{l}
n \\
4
\end{array}\right)-N(3)-N(5)}(1-(q-2) t)^{\left(\begin{array}{c}
n \\
2
\end{array}\right)-N(3)}(1-(q-1) t)^{-n+\left[\frac{k}{q-1}\right]+2}
\end{aligned}
$$


and

$$
P_{2}(t)=(1-(q-1) t)^{-\left[\frac{k}{q-1}\right]-2}(1-q t)
$$

$P_{2}(t)$ has positive coefficients because of Lemma 14 .

For the positivity of $P_{1}(t)$, we define

$$
E(r):=\left(\begin{array}{l}
n \\
r
\end{array}\right)-N(r-1)-N(r+1) .
$$

Then there is an even integer $q_{0}$ such that $q_{0}<\frac{n}{2}-\frac{\sqrt{n}}{2}$, and

$$
E(r) \leq 0 \text { if and only if } r \leq q_{0} .
$$

If $q-2 \leq q_{0}$, then $P_{1}(t)$ is of the form $\frac{1}{\Pi\left(1-a_{i} t\right)^{n_{i}}}$ and we are done.

If $q-2>q_{0}$ and $q \geq 6$, then

$$
P_{1}(t)=\frac{(1-2 t)^{E(q-2)}(1-4 t)^{E(q-4)} \cdots\left(1-\left(q-q_{0}-2\right) t\right)^{E\left(q_{0}+2\right)}}{\left(1-\left(q-q_{0}\right) t\right)^{-E\left(q_{0}\right)} \cdots(1-(q-4) t)^{-E(4)}(1-(q-2) t)^{N(3)-\left(\begin{array}{c}
n \\
2
\end{array}\right)(1-(q-1) t)^{n-\left[\frac{k}{q-1}\right]-2}} .} .
$$

According to Proposition 12, the condition for the positivity of $P_{1}(t)$ is

$$
\sum_{q_{0}+2 \leq \underset{\text { even }}{r} \leq q-2} E(r) \leq-\sum_{\substack{r \leq r \leq n \\
\text { even }}} E(r)+N(3)-\left(\begin{array}{l}
n \\
2
\end{array}\right)+n-\left[\frac{k}{q-1}\right]-2
$$

i.e.

$$
\sum_{4 \leq \underset{\text { even }}{r} \leq q-2} E(r) \leq N(3)-\left(\begin{array}{l}
n \\
2
\end{array}\right)+n-\left[\frac{k}{q-1}\right]-2 .
$$

According to (19) and (21), it suffices to show

$$
\begin{aligned}
\sum 2 & \left(\begin{array}{l}
n \\
r
\end{array}\right)-\left(\begin{array}{c}
n \\
r-1
\end{array}\right)-\left(\begin{array}{c}
n \\
r+1
\end{array}\right) \\
& +\frac{(n-1)}{\sqrt{2}}\left\{\frac{1}{q-r+1}\left(\begin{array}{c}
n \\
r-1
\end{array}\right)^{1 / 2}+\frac{1}{q-r-1}\left(\begin{array}{c}
n \\
r+1
\end{array}\right)^{1 / 2}\right\}+q-2 \\
& \leq\left(\begin{array}{c}
n \\
3
\end{array}\right)-\frac{(n-1)}{\sqrt{2}(q-3)}\left(\begin{array}{c}
n \\
3
\end{array}\right)^{1 / 2}-2\left(\begin{array}{l}
n \\
2
\end{array}\right) .
\end{aligned}
$$

Using Lemma 15 (i), we see that it suffices to show

$$
\begin{gathered}
\frac{(n-1)}{\sqrt{2}}\left\{\frac{1}{q-3}\left(\begin{array}{c}
n \\
3
\end{array}\right)^{1 / 2}+\sum_{4 \leq \text { even } \leq q-2} \frac{1}{q-r+1}\left(\begin{array}{c}
n \\
r-1
\end{array}\right)^{1 / 2}\right. \\
\left.+\frac{1}{q-r-1}\left(\begin{array}{c}
n \\
r+1
\end{array}\right)^{1 / 2}\right\} \\
\leq\left(\begin{array}{c}
n-1 \\
q-1
\end{array}\right)-\left(\begin{array}{c}
n-1 \\
q-2
\end{array}\right)-q-2\left[\frac{n-1}{q-1}\right] .
\end{gathered}
$$

Since $q-1 \leq \frac{n}{2}$, on the left hand side of the inequality the largest binomial coefficient is $\left(\begin{array}{c}n \\ q-1\end{array}\right)$. It suffices to replace the left hand side by

$$
\frac{(n-1)}{\sqrt{2}}\left(\begin{array}{c}
n \\
q-1
\end{array}\right)^{1 / 2}\left(1+\frac{2}{3}+\frac{2}{5}+\cdots+\frac{2}{q-5}+\frac{2}{q-3}\right) .
$$


It follows from Lemma 16 that it suffices to show

$$
\begin{aligned}
\frac{n-1}{\sqrt{2}}\left(\begin{array}{c}
n \\
q-1
\end{array}\right)^{1 / 2} \frac{(q+25)}{14}+q+2 \frac{(n-1)}{q+1} & \leq\left(\begin{array}{c}
n-1 \\
q-1
\end{array}\right)-\left(\begin{array}{c}
n-1 \\
q-2
\end{array}\right) \\
& =\frac{(n-1) !}{(q-1) !(n-q+1) !}(n-2 q+2) .
\end{aligned}
$$

On the other hand, for $\frac{n}{2}-1 \geq q \geq 6$,

$$
\frac{q}{n-1}+\frac{2}{q+1}<\frac{9}{10}<\frac{1}{14 \sqrt{2}}\left(\begin{array}{c}
n \\
q-1
\end{array}\right)^{1 / 2} .
$$

So after dividing by $n-1$, it suffices to show

$$
\left(\begin{array}{c}
n \\
q-1
\end{array}\right)^{1 / 2} \frac{(q+26)}{14 \sqrt{2}} \leq \frac{(n-2) !}{(q-1) !(n-q+1) !}(n-2 q+2) .
$$

After squaring both sides and simplifying, we have

$$
\frac{n(n-1)(q+26)^{2}}{2 \cdot 14^{2}} \leq \frac{(n-2) !(n-2 q+2)^{2}}{(q-1) !(n-q+1) !}
$$

i.e.

$$
1 \leq \frac{49(n-2)(n-3) \cdots(n-q+2)(n-2 q+2)^{2}}{n(n-1)(q+26)^{2}(q-1)(q-2) \cdots 6 \cdot 5 \cdot 3} .
$$

Our assumption $n \geq 2 q+2$ gives

$$
\frac{n}{2}+25 \geq q+26
$$

Therefore it suffices to show

$$
1 \leq \frac{4 \cdot 49(n-2) \cdots(n-q+2)(n-2 q+2)^{2}}{n(n-1)(n+50)^{2}(q-1) \cdots 6 \cdot 5 \cdot 3} .
$$

Now (28) follows from

$$
\frac{(n-2)(n-3) \cdots(n-6) \cdot 3}{n(n-1)(n+50)^{2}}>1, \text { if } n \geq 18,
$$

and

$$
\frac{4 \cdot 49(n-7)(n-8) \cdots(n-q+2)(n-2 q+2)^{2}}{3(q-1)(q-2) \cdots 7 \cdot 6 \cdot 5 \cdot 3}>1, \text { if } q \geq 9
$$

The last inequality holds, because it is at least

$$
\frac{n-q+2}{12} \frac{12 \cdot 4 \cdot 49(n-2 q+2)^{2}}{3 \cdot 8 \cdot 7 \cdot 6 \cdot 5 \cdot 3}
$$

and

$$
\frac{12 \cdot 4 \cdot 49}{3 \cdot 8 \cdot 3 \cdot 3 \cdot 3}(n-2 q+2)^{2}>\frac{(n-2 q+2)^{2}}{4^{2}}>1
$$

For $q=8$ (respectively 6 ), we check directly that for $n \geq 18$ (resp. 14) inequality (28) (resp. (24)) holds. 
(ii) For $p \geq 7$, odd, we replace (6) by $\left(6^{\prime}\right)$, and (20), (22), (23), (24), (25), (27) and (28) by

$$
\frac{(1-2 t)^{N(q-1)}}{(1-t)^{\left(\begin{array}{c}
n \\
q-1
\end{array}\right)}} \frac{[(1-2 t)(1-4 t)]^{N(q-3)}}{(1-3 t)^{\left(\begin{array}{c}
n \\
q-3
\end{array}\right)}} \cdots \frac{[(1-(q-3) t)(1-(q-1) t)]^{N(2)}}{(1-(q-2) t)^{\left(\begin{array}{c}
n \\
2
\end{array}\right)}} \cdot P(t),
$$

where

$$
\begin{aligned}
P(t) & =(1-2 t)^{E(q-2)} \cdots(1-(q-3) t)^{E(3)}(1-(q-1) t)^{n-N(2)}(1-q t)^{-1} \\
& =\frac{(1-2 t)^{E(q-2)}(1-4 t)^{E(q-4)} \cdots\left(1-\left(q-q_{0}-2\right) t\right)^{E\left(q_{0}+2\right)}}{\left(1-\left(q-q_{0}\right) t\right)^{-E\left(q_{0}\right)} \cdots(1-(q-3) t)^{-E(3)}(1-(q-1) t)^{N(2)-n}(1-q t)},
\end{aligned}
$$

$$
\begin{aligned}
& \sum_{\substack{r \\
\text { odd } \leq q-2}} E(r) \leq N(2)-n+1, \\
& q-5+\frac{(n-1)}{\sqrt{2}}\left\{\frac{1}{q-2}\left(\begin{array}{l}
n \\
2
\end{array}\right)^{1 / 2}+\sum_{3 \leq r \leq q-2} \frac{1}{q-r+1}\left(\begin{array}{c}
n \\
r-1
\end{array}\right)^{1 / 2}\right. \\
& \left.+\frac{1}{q-r-1}\left(\begin{array}{c}
n \\
r+1
\end{array}\right)^{1 / 2}\right\} \\
& \leq\left(\begin{array}{l}
n-1 \\
q-1
\end{array}\right)-\left(\begin{array}{l}
n-1 \\
q-2
\end{array}\right) \\
& \left(\begin{array}{c}
n \\
q-1
\end{array}\right)^{1 / 2} \frac{(q+27)}{14 \sqrt{2}} \leq \frac{(n-2) !}{(q-1) !(n-q+1) !}(n-2 q+2), \\
& 1 \leq \frac{49(n-2)(n-3) \cdots(n-q+2)(n-2 q+2)^{2}}{n(n-1)(q+27)^{2}(q-1) \cdots 6 \cdot 5 \cdot 3}, \\
& 1 \leq \frac{4 \cdot 49(n-2) \cdots(n-q+2)(n-2 q+2)^{2}}{n(n-1)(n+52)^{2}(q-1) \cdots 6 \cdot 5 \cdot 3} .
\end{aligned}
$$

For $q=7$, we check directly that inequality $\left(27^{\prime}\right)$ holds for $n \geq 16$.

(iii) For $q=4$, we write (6) as

$$
\frac{(1-2 t)^{\left(\begin{array}{c}
n \\
2
\end{array}\right)-n+N-1}}{(1-t)^{\left(\begin{array}{c}
n \\
3
\end{array}\right)}} \frac{(1-2 t)^{n-N}}{(1-3 t)^{n-N}} \frac{(1-2 t)(1-4 t)}{(1-3 t)^{N}} .
$$

Here $N=\left[\frac{n}{3}+\frac{4}{n}\right]+1$.

Proposition 10 and Lemma 11 imply positive coefficients for $n \geq 11$. For $n=10$, one can easily check by hand.

For $q=5$, we write $\left(6^{\prime}\right)$ as

$$
\frac{(1-2 t)^{N_{2}}}{(1-t)^{\left(\begin{array}{c}
n \\
4
\end{array}\right)}}\left(\frac{1-2 t}{1-3 t}\right)^{\left(\begin{array}{c}
n \\
2
\end{array}\right)-M_{1}} \frac{[(1-2 t)(1-4 t)]^{n-1}}{(1-3 t)^{M_{1}}} \frac{1-4 t}{1-5 t} .
$$


Here

$$
M_{1}=\left[2(n-1)+\frac{\sqrt{n-2}}{3}(n-1)\right]+1
$$

and

$$
N_{2}=\left(\begin{array}{l}
n \\
3
\end{array}\right)-\left(\begin{array}{l}
n \\
2
\end{array}\right)+M_{1}-n+1
$$

Proposition 10 and Lemma 11 imply positivity for $n \geq 12$.

Lemma 14. The coefficient of $t^{k}$ in

is positive, if

$$
\frac{1-q t}{(1-(q-1) t)^{M}}, \quad q>1
$$

$$
(M-1)(q-1)>k .
$$

Proof. We write $(1-(q-1) t)^{-M}$ as

$$
\sum\left(\begin{array}{c}
M+i-1 \\
i
\end{array}\right)(q-1)^{i} t^{i}
$$

The coefficient of $t^{k}$ is

$$
\left(\begin{array}{c}
M+k-1 \\
k
\end{array}\right)(q-1)^{k}-\left(\begin{array}{c}
M+k-2 \\
k-1
\end{array}\right)(q-1)^{k-1} q,
$$

which is

$$
(q-1)^{k} \frac{(M+k-2) !}{k !(M-1) !}[(M-1)(q-1)-k]
$$

Remark 14.1. Similar to the argument in Proposition 10, one can show that if $c(N, M, k)$ is the coefficient of $t^{k}$ in

$$
\frac{(1-a t)^{N}}{(1-b t)^{M}}, \quad a>b
$$

then

$$
0<c(N, M, k)<b^{k}\left(\begin{array}{c}
M-N+k-1 \\
k
\end{array}\right),
$$

if $b(M-N)>(a-b) N K$.

Lemma 15. (i) $\sum_{4 \leq r<\leq e-2} 2\left(\begin{array}{l}n \\ r\end{array}\right)-\left(\begin{array}{c}n \\ r-1\end{array}\right)-\left(\begin{array}{c}n \\ r+1\end{array}\right)=\left(\begin{array}{l}n-1 \\ q-2\end{array}\right)-\left(\begin{array}{l}n-1 \\ q-1\end{array}\right)+$ $\left(\begin{array}{c}n-1 \\ 3\end{array}\right)-\left(\begin{array}{c}n-1 \\ 2\end{array}\right)$

$$
\text { (ii) } \sum_{3 \leq r \leq q-2} 2\left(\begin{array}{c}
n \\
r
\end{array}\right)-\left(\begin{array}{c}
n \\
r-1
\end{array}\right)-\left(\begin{array}{c}
n \\
r+1
\end{array}\right)=\left(\begin{array}{c}
n-1 \\
q-2
\end{array}\right)-\left(\begin{array}{c}
n-1 \\
q-1
\end{array}\right)+2 \text {. }
$$

Proof. Same as Lemma 7.

Lemma 16. $2\left(\frac{1}{3}+\frac{1}{5}+\cdots+\frac{1}{m-2}+\frac{1}{m}\right)<1+\frac{m}{14}$. 
Proof. It is easy to see that by induction if the inequality holds for $m>12$, then it holds for $m+2$. The initial steps for $m \leq 12$ can be done by hand.

Remark 16.1. The expression on the right hand side is obtained by bounding $\left(\frac{1}{3}+\right.$ $\left.\frac{1}{5}\right)+\left(\frac{1}{7}+\cdots+\frac{1}{m}\right)$ by $\frac{16}{15}+\left(\frac{1}{7}+\frac{1}{m}\right)\left(\frac{m-5}{2}\right)$, and rounding it off.

Lemma 17. The coefficient of $t^{k}$ is positive for $k \leq n-1$ in the following functions.

(i) $(p=1$ case $) \frac{1}{1-t}$.

(ii) $\left(p=2\right.$ case) $\frac{1-2 t}{(1-t)^{n}}$, except for $k=n-1$.

(iii) $(p=3$ case $) \frac{(1-2 t)^{n}}{(1-t)\left(\begin{array}{c}n \\ 2\end{array}\right)(1-3 t)}$.

Proof. (i) is obvious. Case (ii) follows from Proposition 10. For case (iii), we apply Proposition 10 and Lemma 11 to $\frac{(1-2 t)^{n-1}}{(1-t)^{\left(\begin{array}{c}n \\ 2\end{array}\right)}} \frac{(1-2 t)}{(1-3)}$.

\section{REFERENCES}

[BH] Barth, W., Hulek, K., Monads and moduli of vector bundles, Manuscripta Math. 25 (1978), 323-347. MR 80f:14005

[BG] Bloch, S., Gieseker, D., The positivity of the Chern classes of an ample vector bundle, Inv. Math 12 (1971), 112-117. MR 45:6825

[C] Chang, M-C, Some remarks on Buchsbaum bundles, J. Pure Appl. Algebra 152 (2000), 1-3. MR 2001f: 14082

[E1] Ein, L., Generalized null correlation bundles, Nagoya Math. J. 111 (1988), 13-24. MR 89k:14024

[E2] Ein, L., Private communication.

[EHS] Elencwajg, G., Hirschowitz, A., Schneider, M., Les fibres uniformes de rang au plus $n$ sur $\mathbb{P}_{n}(\mathbb{C})$ sont ceux qu'on croit, Proceedings of the Nice conference on vector bundles and differential equations, (1979). Birkhäuser, Boston, 1980. MR 81k:14015

[FL] Fulton, W., Lazarsfeld, R., Positive polynomials for ample vector bundles, Annals of Math. 118, (1983), 35-60. MR 85e:14021

[H1] Hartshorne, R., Algebraic geometry, GTM 152, Springer-Verlag, Heidelberg and New York, (1977). MR 57:3116

[H2] Hartshorne, R., Algebraic vector bundles on projective spaces: A problem list, Topology 18, (1979), 117-128. MR 81m:14010

[H3] Hartshorne, R., Varieties of small codimension in projective spaces, Bull. A.M.S. 80, (1974), 1017-1032. MR 52:5688

[Ho] Horrocks, G., Examples of rank three vector bundles on five-dimensional projective space, J. London Math. Soc. (2) 18 (1978), 12-27. MR 80d:14011

[HM] Horrocks, G., Mumford, D., A rank 2 vector bundle on $\mathbb{P}^{4}$ with 15,000 symmetries, Topology 12, (1973), 63-81. MR 52:3164

[KPR] Kumar, M., Peterson, C., Rao, P., Construction of low rank vector bundles on $\mathbb{P}^{4}$ and $\mathbb{P}^{5}$ (preprint).

[KS] Katz, S., Stromme, S., A Maple package for intersection theory.

[LV] Lazarsfeld, R., Van de Ven, A., Topics in the Geometry of Projective Spaces, DMV Sem. 4, Birkhäuser Verlag, Basel and Boston, (1984). MR 87e:14045

[OSS] Okonek, C., Schneider, M., Spindler, H., Vector bundles on Complex Projective Spaces, Progress in Mathematics, 3 Birkhäuser, Boston, VIII (1980). MR 81b:14001

[T] Tango, H., An example of indecomposable vector bundles of rank $n-1$ on $\mathbb{P}_{n}$, J. Math. Kyoto Univ. 16 (1976), 137-141. MR 53:5593

[V] Vogelaar, J.A., Constructing vector bundles from codimension - two subvarieties, Thesis, Leiden 1978.

[Z] Zak, F., Tangents and secants of algebraic varieties, AMS Transl. Math. Monos., 127 (1993). MR 94i:14053

Department of Mathematics, University of California, Riverside, California 92521

E-mail address: mcc@math.ucr.edu 